Beinert ${ }^{5}$ has investigated the spectrum of riboflavin as it goes from the oxidized to the reduced form. The absorption spectrum of oxidized riboflavin has peaks at 375 and $445 \mathrm{~nm}$ whereas the reduced riboflavin only has a peak at $251 \mathrm{~nm}$ and has less absorption than the oxidized form. There is an isobestic point in the spectrum at $330 \mathrm{~nm}$. Beinert has concluded that the spectrum of riboflavin is due to the sum of three components, the oxidized form, the reduced form, and an intermediate form, the semiquinone.

The difference spectra observed in amino-acid-riboflavin mixtures at different $p \mathrm{H}^{\prime}$ s can best be explained by the stabilization of the semiquinone form of riboflavin by the amino-acid. Isenberg and Szent-Györgyi ${ }^{3}$ have suggested that the tryptophan riboflavin- $\breve{s}^{\prime}$-phosphate interaction can be explained by the tryptophan forming a $\pi-\pi$ charge-transfer complex with the riboflavin-5'-phosphate thus:

tryptophan + riboflavin $-5^{\prime}$-phosphate $\rightleftharpoons$ (oxidized tryptophan-semiquinone $)$ complex

Such a scheme could well describe the interaction of the amino-acids with riboflavin, except that the donated electron reducing the riboflavin should be a lone-pair $(n)$ electron, probably from the amino-group. There appears no reason why only tryptophan should interact with riboflavin $-5^{\prime}$-phosphate to give a $\pi-\pi$ complex, whereas the amino-acids as a whole react with other quinones such as chloranil and riboflavin in such a manner that an $n$-electron is transferred from the amino-acid to the quinone.

The tenure of a Department of Scientific and Industrial Research fellowship and research studentship is acknowledged.

\section{A. SLIFKIN}

Physical Laboratories,

University of Manchester.

${ }^{1}$ Slifkin, M. A., Nature, 193, 464 (1962).

${ }^{2}$ Birks, J. B., and Slifkin, M. A., Nature, 197, 42 (1963). ${ }^{3}$ Isenberg, I., and Szent-Györgyi, A., Proc. U.S. Nat. Acad. Sci., 44, 857
(1958).

+ Weber, G., Biochem. J., 47, 114 (1950).

s Beinert, M., J. Amer. Chem. Soc., 78, 5323 (1956).

\section{GEOPHYSICS}

\section{Formation of Mountains by Radial Contraction of the Earth}

Modern ideas on the origin of the planets suggest that the Earth began as a cool solid body, and on this basis the development of a liquid core, of gradually increasing size, would be ascribed to subsequent radioactive heating.

The elastic constants of the liquid core and of the solid mantle for the actual Earth are known, and these imply that the core-material, although liquid, is more compressible than the mantle-material at the pressures prevail. ing deep within the Earth. Making use of the known values, the properties of a linear series of Earth-models of gradually increasing core-mass can be investigated, beginning with an entirely solid Earth. Calculations show that the radius decreases as the core-mass increases, and that an initial solid Earth would have outer radius 1.043 times the present value $R_{E}$, while allowance for the lower uncompressed density of the material of the outer layers would increase this figure to $1.056 R_{E}$. This result would mean an initial circumferential length more than $2,000 \mathrm{~km}$ greater than the present value, and an initial surface-area exceeding by nearly 60 million square kilometres the present area of the Earth.

The importance of such a contraction of the Earth as a whole to the problem of the formation of mountains is evident, since it would gradually build up shearing stresses in the outer layers that the materials there would eventually be unable to withstand, and periods of folding and thrusting would have to occur intermittently to relieve the stresses. The mechanism appears to be fully capable of providing the seemingly enormous amounts of contraction that have long been considered necessary by geologists to explain the actual mountain systems on Earth.

The mathematical theory and detailed calculations leading to these conclusions are in course of publication.

\section{R. A. Lyttleton}

St. John's College, Cambridge.

\section{GEOCHEMISTRY}

\section{Low Concentration of Tritium in Iron Meteorites}

A sErIous geochemical problem that is not yet solved is the quantity of tritium found in iron meteorites. Although all other isotopes produced by cosmic rays in both iron and stone meteorites are in close agreement with expected values estimated by cross-section measurements using large accelerators ${ }^{1}$, tritium is either absent or at least an order of magnitude less than expected in iron meteorites (for example, ref. 2, and the references listed in that article). The tritium content of stone meteorites is not abnormal.

Two explanations have been proposed for the low concentration of tritium in iron meteorites. It has been suggested that the chemical treatment methods are inefficient and the tritium is not extracted from the iron. This, however, seems difficult to believe since the two different chemical methods used ${ }^{2,3}$ are both reliable and completely attack the iron. Furthermore, the hydrogen gas added as carrier and dissolved in the iron is always recovered, quantitatively.

The other explanation proposed is that tritium diffuses out of iron meteorites. However, direct diffusion measurements of tritium in iron do not agree with this and the ratio of helium-3 to helium-4 in iron meteorites is in close agreement with that predicted from accelerator measurements ${ }^{4}$. This requires that the tritium formed in the meteorites during the flights in space must have remained in the meteorites and decayed to helium.3. Another suggestion is that a special mechanism permits the tritium to diffuse out of the iron meteorites only after they have reached the Earth. At present, there is no plausible theory that would predict this.

Further evidence supporting the diffusion loss hypothesis is the observation of what is called a diffusion of tritium out of iron acceleration targets ${ }^{5,6}$. However, an examination of the experiments reveals that diffusion was not directly observed, but merely the phenomenon that the longer the iron targets remained untreated after the irradiations, the less tritium was extracted. It is noted that this is not observed in other metal targets.

Since neither of the two aforementioned hypotheses seems reasonable, the tritium content of iron meteorites is now an important mystery for science and, therefore, all sorts of possibilities should be checked. I suggest here the possibility that a completely new phenomenon is being observed. That is, that tritium dissolved in iron, possibly denuded of its electrons and held in a strong magnetic field, no longer decays with its ordinary 12-years half-life, but much more rapidly, perhaps with a half-life of the order of several days or even less. It seems that both a combination of a nucleus without an ordinary electron cloud and the presence of a magnetic field would be necessary.

An experiment is possible to test this proposition. A stack of iron foils irradiated in an accelerator or otherwise concentrated with dissolved tritium could be submitted, 